\title{
Multiple Small Bowel Atresia: Resection or Conservation?
}

\author{
Krishna Kumar Govindarajan ${ }^{1}$ and Mohanaprakash Annamalai ${ }^{2}$ \\ ${ }^{1}$ Department of Pediatric Surgery, JIPMER, India \\ ${ }^{2}$ Department of Surgery, HTAA, Malaysia
}

\begin{abstract}
Multiple small bowel atresia is a relatively uncommon cause of intestinal obstruction in neonates. About one-third of the neonatal intestinal obstruction is caused by intestinal atresia. As suggested by Louw and Barnard, the vascular accident in utero is the principal reason, resulting in bowel atresia. ${ }^{1}$ The condition presents soon after birth, with bilious vomiting and abdominal distension, requiring emergency surgical intervention. A subtype of small bowel atresia (type IV), which presents with involvement of multiple segments, is unique due to the difficulties in management, accompanied by a variety of postoperative morbidities, which are highlighted in this case. The choice lies between resection of all the atretic segments with resultant single anastomosis and conservation with anastomoses of multiple small bowel segments involving meticulous repair by fine sutures, taking care to maintain luminal patency. The dilemma of whether to conserve the atretic segments of bowel, which is a time consuming procedure but preserves the bowel length or to proceed with excision of the atretic bowel segments, accepting the likelihood of short bowel, in order to provide a quick, safe and stable single anastomosis in a timely manner, is a valid challenge. The background of limited resource setting also need to be taken into consideration.
\end{abstract}

Key Words: Atresia, Neonates, Bowel segments, Anastomosis.

How to cite this article: Govindarajan KK, Annamalai M. Multiple Small Bowel Atresia: Resection or Conservation?. J Coll Physicians Surg Pak 2021; 31(06):740-742.

A male baby was delivered prematurely at 34 weeks of gestation with birth weight of $1.9 \mathrm{~kg}$. Antenatal history was uneventful. On day-3 of life, he was not tolerating feeds with increasing greenish aspirates, and worsening bowel dilation on plain abdominal x-ray (Figure 1). He was subjected to laparotomy for small bowel atresia, after informed written consent from the parents. Intraoperatively, three atretic small bowel segments, each varying from $6-10 \mathrm{~cm}$, were noted in between $20 \mathrm{~cm}$ from duodenojejunal (DJ) flexure and $25 \mathrm{~cm}$ from ileocaecal (IC) junction (Figure 2). A decision of resection with primary anastomosis, leaving about $45 \mathrm{~cm}$ of small bowel, was undertaken to shorten the operating time, keeping in mind the preterm status of the baby. Postoperatively, the baby developed multiple episodes of diarrhoea, when initiated on full oral feeds. Together with this, he was noted to have poor weight gain, prompting parenteral nutrition. He weighed $6.8 \mathrm{~kg}$ at 11 months of age. During the course of parenteral nutrition, he developed episodes of central venous line related sepsis and cholestasis, prompting frequent change of central venous access.

Correspondence to: Dr. Krishna Kumar Govindarajan, Department of Paediatric Surgery, JIPMER, India

E-mail: kkpeds@gmail.com

Received: July 27, 2020; Revised: August 28, 2020;

Accepted: September 08, 2020

DOI: https://doi.org/10.29271/jcpsp.2021.06.740
The episodes of diarrhoea persisted and were attributed to short bowel syndrome, which was managed with antimotility agent, loperamide. Due to all the above mentioned issues (short bowel, parenteral nutrition, failure to thrive), he was not discharged and continued a prolonged in-hospital care. Over the next few months, he was weaned off parenteral nutrition and antimotility agent. By 15 months, he weighed $8.6 \mathrm{~kg}$ and was able to tolerate full oral feeds with passage of formed stools. At two year follow-up, the child was at $5 \%$ of expected weight $(9.3 \mathrm{~kg})$.

A second baby, a term child delivered with birth weight of $2.6 \mathrm{~kg}$, developed abdominal distension at nine hours after birth and multiple episodes of bilious vomit. The plain abdominal Xray showed dilated bowel loops, suggestive of bowel atresia and was subjected to laparotomy after written consent. Intraoperatively, four atretic small bowel segments were identified in between $32 \mathrm{~cm}$ from DJ flexure and $12 \mathrm{~cm}$ from IC junction (Figure 3). The smallest atretic segment measuring $8 \mathrm{~cm}$ was excised and the other three segments measuring $10-15 \mathrm{~cm}$ were joined by multiple anastomosis (three) resulting in conservation of $92 \mathrm{~cm}$ of small bowel. The baby withstood the prolonged operative procedure. Postoperatively, the child was maintained on parenteral nutrition for three weeks. After a upper gastrointestinal contrast study to evaluate the transit time through the multiple small bowel anastomoses, he was gradually introduced to enteral feeding, taking about 10 days to establish full oral feeds. On recent follow-up at 18 months, the baby had good weight gain $(10.2 \mathrm{~kg})$ with no futher complications. 


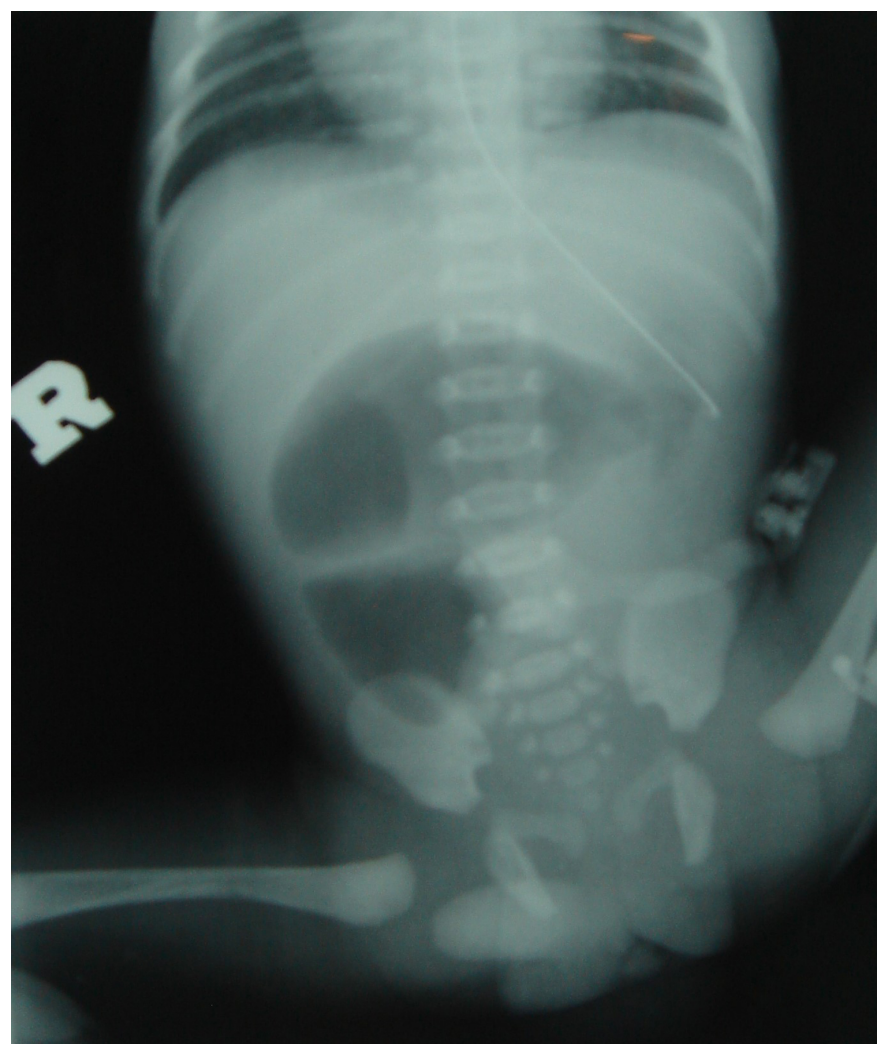

Figure 1: Plain X-ray abdomen - Multiple, dilated air-fluid levels suggestive of intestinal obstruction.

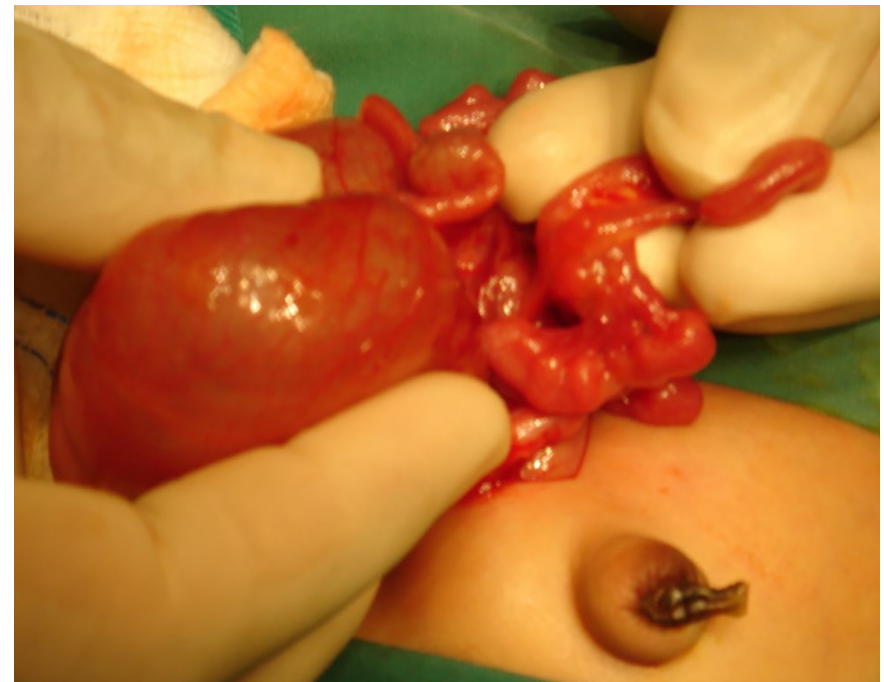

Figure 2: Intraoperative photograph demonstrating multiple small bowel atretic segments in case 1.

The incidence of jejunoileal atresia is reported to be around 1 in 300 live births. Atresia of the small bowel is classified into 4 categories, with the type IV atresia being the rarest, accounting for only $6 \%$ of all jeunoileal atresias. Although the occurence of type IV is rare, the treament modalities pose a dilemma for surgeons. On the one hand, excision of all atretic segments with one single anastomosis, as in case 1 , would reduce the risk of anastomotic complications such as leak, stricture and delayed establishment of full oral feeds. But then, the risk of short bowel syndrome with prolonged usage of parenteral nutrition with its associated problems, namely line related sepsis and choles- tasis need to be kept in mind. Short bowel can be a difficult problem owing to the challenge of providing the appropriate nutrition within the range of bowel absorption and waiting out on the bowel adaptation, which can be prolonged upto 1-2 years post-bowel resection. ${ }^{2}$ On the other hand, although the risk of anastomotic leakage and stricture is higher in multiple anastomoses, the outcome of the patient in long-term seems to be more favourable. ${ }^{1}$ Also, the intraoperative time is prolonged when attempting conservation with multiple anastomoses. As per Stollman et al, advancements of treatment modalities for the bowel atresia have brought down the mortality; the rate of morbidity has been on the rise. ${ }^{3}$ The challenge in a limited resource setting is the provision of parenteral nutrition and avoidance of line sepsis. In addition, provision of intensive care, prolonged in-hospital stay and neonatal anesthesia are other contributory factors challenging the limited resource setting. The marked contrast in the postoperative course of both the children shows that multiple anastomoses with conservative bowel resection may be superior to the complete resection of atretic bowel segments with loss of precious length of bowel and consequent short gut syndrome. ${ }^{2,4}$

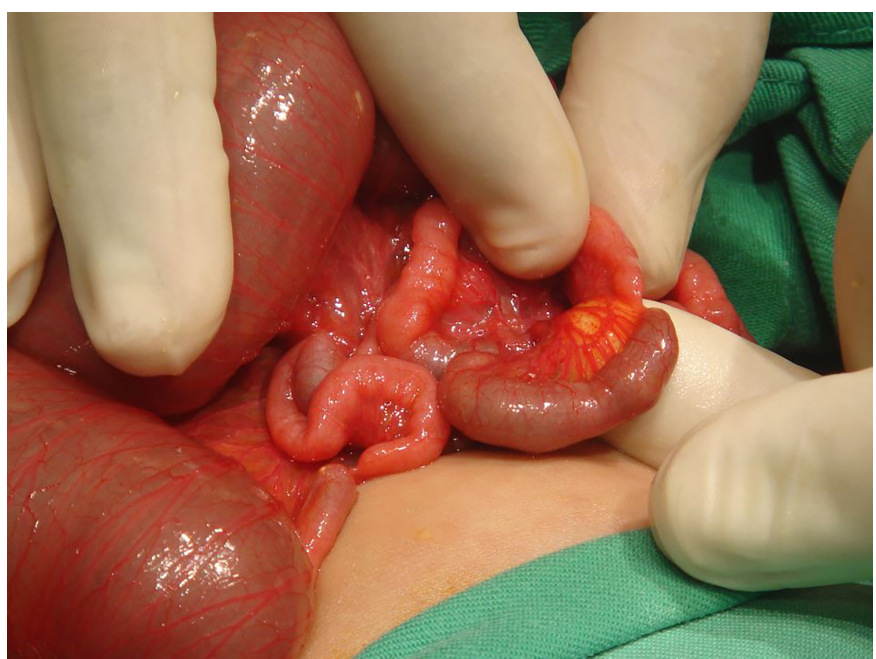

Figure 3: Intraoperative photograph demonstrating multiple small bowel atretic segments in case 2 .

Multiple small bowel atresia is an uncommon but challenging cause of neonatal intestinal obstruction. Weighing the pros and cons between conservation and resection, the preferred surgical option should be to undertake multiple anastomoses of the small bowel segments resulting in preservation of gut length with good outcome in the long-term, provided the child can withstand the prolonged operating time.

\section{CONFLICT OF INTEREST:}

The authors declared no conflict of interest.

\section{AUTHORS' CONTRIBUTION:}

KKG: Conception, final revision.

MA: Drafting of manuscript.

\section{REFERENCES}

1. Sato S, Nishijima E, Muraji T, Tsugawa C, Kimura K. Jejunoileal atresia: A 27-year experience. J Pediatr Surg 
1998; 33(11):1633-5. doi: 10.1016/s0022-3468(98) 90596-6.

2. Chandra R, Kesavan A. Current treatment paradigms in pediatric short bowel syndrome. Clin J Gastroenterol 2018; 11(2):103-12. doi: 10.1007/s12328-017-0811-7

3. Stollman $\mathrm{TH}$, de Blaauw I, Wijnen $\mathrm{MH}$, van der Staak FH, Rieu PN, Draaisma JM, et al. Decreased mortality but increased morbidity in neonates with jejunoileal atresia; a study of 114 cases over a 34-year period. J Pediatr Surg
2009; 44(1):217-21. doi: 10.1016/j.jpedsurg.2008.10.043.

4. Sapin E, Carricaburu E, De Boissieu D, Goutail-Flaud MF, Benammar S, Helardot PG. Conservative intestinal surgery to avoid short-bowel syndrome in multiple intestinal atresias and necrotizing enterocolitis: 6 cases treated by multiple anastomoses and Santulli-type enterostomy. Eur J Pediatr Surg 1999; 9(1):24-8. doi: 10.1055/s-20081072207. 